\title{
The Three-dimensional Complex Space Theory
}

\author{
Daniel Eduardo Caminoa Lizarralde ${ }^{1}$ \\ ${ }^{1} 78$ Herrera y Guzman Street, Cordoba X5008CZB, Argentina \\ Correspondence: Daniel Eduardo Caminoa Lizarralde, 78 Herrera y Guzman Street, Cordoba X5008CZB, \\ Argentina. Tel: 54-035-1477-0533. E-mail: dcaminoa@gmail.com
}

Received: December 13, 2011

Accepted: December 26, 2011 Online Published: May 1, 2012

doi:10.5539/apr.v4n2p190

URL: http://dx.doi.org/10.5539/apr.v4n2p190

\begin{abstract}
This work expands of the paper The Three-dimensional Complex Space theory - TCS Theory (Caminoa Lizarralde Daniel E., 2009 and 2010) with the advances obtained in TCS Theory - Planetary Mechanics TCS PM $_{1}$ (Caminoa Lizarralde Daniel E., 2011). This research was started almost twenty-four years ago at Buenos Aires. Two experiments were selected on which I carry out interpretations of this new theory. Also, I've added a complete development study on electromagnetic hysteresis, due to it is of primary importance on astronomical research the determination of new electromagnetic spectra that happen on the Cosmos and it is at range of observation. The TCS theory determines an existence of six different quarks (only two of them can be observed with present electromagnetic technologies), as well as three elemental particles components that integrates every known matter and it belongs to three different physical subspaces (even temporal subspace), that makes nine physical dimensions and twelve real dimensions in total.
\end{abstract}

Keywords: physical space, subspaces, absolute time, matter, quarks, atomic particles, subspaces waves, electromagnetic hysteresis

\section{Introduction}

This research have a remarkable consequence because it involves knowledge about the space developed by great scientists, Euclid of Alexandria, Plato, René Descartes, Sir Isaac Newton, Christian Huygens, Max Karl Ernst Ludwig Planck, Georges-Louis Le Sage, James Clerk Maxwell, Hendrik Antoon Lorentz, Albert Einstein, David Hilbert as well others, all of them gave a substantial contribution into the TCS theory, as we can see in the next steps.

The following paragraphs describe a new interpretation of the real space or the cosmological space, maintaining the three-dimensional Euclidean space and incorporating in its essence for the first time the concept of "subspace"; this new and complex multidimensional cosmological conception allows us to be in absolutely agreement with the mathematical postulates of Giuseppe Peano (1889) and David Hilbert (1899) of the mathematics and the formal logic, whose more immediate consequence and primordial is that we can move or rotate the coordinate in the physical equations without altering the Laws involved. This fundamental property determines the universality and immutability of natural laws of science in space and time (in the Universe).

In other multidimensional cosmological conceptions (4 or more dimensions) where they do not agree the principle of dimensional homogeneity -such as using non-homogeneous coordinates, mixing in a unique dimensional space, the dimensions of time with the dimensions of space or others-makes unusual results, away from formal logic and the world we live in.

The two most significant properties of each subspace are the average energy density and elasticity of the field. These two properties make possible the existence of quarks and waves of each subspace. The elasticity of each subspace is not perfect and due to this (hysteresis) is produced the loss of energy on all wave phenomenons.

The universe is unique, of permanent existence and with a volume impossible to establish with current scientific knowledge. The Universe is fragmented into many spheres (Cosmos) of not eternal existence. In one of these Cosmos contains our galaxy (the Milky Way), all galaxies and stars that we can see and Earth, our habitat. All Cosmos has a cyclical existence of millions of eons to determine to existence or not in it's the bosom of the matter and its beings. Every Cosmos suffer periodic cataclysms determined by the subspace density decrease due to the continued expansion and steady increase of matter within it that migrates from the outer layer. After the cataclysmic collapse due to the energy density and elasticity of the subspaces arises rebirth in the form of Big-bang. 


\section{The Subspaces}

The real space is three-dimensional and complex because it is a real three-dimensional space just as Euclid of Alexandria (325/265 BC) did, however, each dimension corresponds with a complex function because it is consisted by four subspaces and each one of them has three elementary dimensions. The real existence into nature is only given for the physical space, in classical physics known as ether or Aether -by ethereal-, it is arranged by four subspaces and by a wave phenomena that happen into this and proper of each subspace. Each one of these subspaces has features and specific constants and it is physically ordered in the nature.

The subspaces are affected with interactions among themselves for every great magnitude phenomenon that happens at astronomic level or due to the effects of nuclear fusion experiments. Hence, the requirement to delay my research about nuclear fusion is due to I believe on the importance to know previously a general review of the three-dimensional complex space theory. The mentioned delay is due to it is necessary to anticipate to any trouble by product of this new energetic technology.

The names that I have assigned to them, the order that they possess and their characteristics are the following ones:

\subsection{Atomic Subspace}

It is the subspace of first order and I have assigned this name because the atomic and nuclear stability of all the natural substances depends on the electric quarks. The electric quarks are wave phenomena of this subspace. The atomic stability is developed in the "Quantum electrodynamics atomic theory" QEDa theory (Caminoa Lizarralde Daniel E, 2005). All the phenomena described by the laws of electromagnetism are within the subspace domain. These phenomena are determined by the interaction of electric quarks that make up atomic particles that exert forces on other quarks of the same domain; the forces are transmitted by alteration and modification of the atomic subspace field that surrounds. All electromagnetism laws within the domain of this subspace was discovered by Charles-Augustin de Coulomb (1736/1806), Johann Carl Friedrich Gauss (1777/1855), Michael Faraday (1791/1867), André-Marie Ampère (1775/1836), Hendrik Antoon Lorentz (1853/1928), Jean-Baptiste Biot (1774/1862) and Félix Savart (1791/1841) and others. Also, within this domain the electromagnetic radiation phenomena are inside the laws of Christiaan Huygens (1629/1695), James Clerk Maxwell (1831/1879), Heinrich Rudolf Hertz (1857/1894), John Henry Poynting (1852/1914), Albert Einstein $(1879 / 1955)$ and others.

These phenomena and their physical constants "Speed of atomic particles and physical constants" $\mathrm{SAPC}_{\mathrm{PC}}$ (Caminoa Lizarralde Daniel E, 2009) are deeply well known nowadays with several publications carried out in the last two centuries.

\subsubsection{Atomic Subspace - Physical Constants}

The system of dimensional units that I use is the IS (International System). It's expressed the electric constant and the magnetic constant respecting classic and old expression.

The values published by NIST are: $c$ Speed of the light is 299792458. m.s ${ }^{-1} ; h$ Planck constant is $6.62606896 \times 10^{-34} \mathrm{~J} . \mathrm{s}$ and $q$ elementary charge is $1.602176487 \times 10^{-19} \mathrm{C}$.

The new values theoretically calculated on $\mathrm{SAP}_{\mathrm{PC}}$ for the remaining physical constants are:

$k_{\mathrm{e}}$ electric constant is $1.23161814398427 \times 10^{12} \mathrm{~N} \cdot \mathrm{m}^{2} \cdot \mathrm{C}^{-2}$;

$k_{\mathrm{m}}$ magnetic constant is $1.37035999694076 \times 10^{-5} \mathrm{~N} \cdot \mathrm{s}^{2} \cdot \mathrm{C}^{-2}$;

$\varepsilon_{0}$ permittivity of free space is $6.46121299321843 \times 10^{-14} \mathrm{~N}^{-1} \cdot \mathrm{m}^{-2} \cdot \mathrm{C}^{2}$;

$\mu_{0}$ permeability of free space is $1.72204515966497 \times 10^{-4} \mathrm{~W} \cdot \mathrm{A}^{-1} \cdot \mathrm{m}^{-1}$;

$Z_{0}$ impedance of free space is $51625.6151202962 \Omega$;

$m_{\mathrm{e}}$ electron inertial mass is $9.09972567274628 \times 10^{-31} \mathrm{~kg}$;

$m_{\mathrm{n}}$ negatron inertial mass is $2.72991770182389 \times 10^{-30} \mathrm{~kg}$ and

$m_{\mathrm{p}}$ proton inertial mass is $1.66979966094894 \times 10^{-27} \mathrm{~kg}$.

Note: In TCS theory there is not the "fine-structure constant" because it would maintain an old error (see $\mathrm{SAP}_{\mathrm{PC}}$; the magnitudes of the constants have the closest absolute value.

The property called "inertial mass" of the atomic particles is a dynamic effect produced jointly by all the quarks by interaction with their subspace domain (inside the atomic subspace, planetary subspace and galactic subspace) determined by the momentum transported by the quarks that compose it. 
The quarks are only waves that carry energy; the energy is a magnitude and the characteristic property of each subspace according to Planck's constant and the unary volume.

\subsection{Planetary Subspace}

It is the subspace of second order and I have assigned this name because the planetary stability of all the planetary systems from the Cosmos depends on the planetary quarks. The planetary quarks are wave phenomena of this subspace. The planetary stability is developed in the "Quantum Electro-Dynamics planetary Theory" QEDp theory that would be published in the future.

All the phenomena described by the gravitomagnetism are within the subspace domain. These phenomena are determined by the interaction of planetary quarks that make up atomic particles that exert forces on other quarks of the same domain; the forces are transmitted by alteration and modification of the planetary subspace field that surrounds. All these phenomena are not scientifically well-known nowadays; only there are carried out some hypotheses about this subject that is denominated gravitomagnetism (GEM) - described for the first time in General Relativity, into the Gravitation Theory published by A. Einstein (1916) and then, by many other scientists- and gravitational waves known within planetary astronomy with considerable consequences regarding the asteroids that cross or belongs to the Kuiper belt.

The laws of gravity by Sir Isaac Newton (1686) can be understood as a brief concept of the QEDp Theory; see $\mathrm{TCS}_{\mathrm{PM}}$ and it will introduce into the laws that govern all planetary systems, specifically in the domain of global stability and satellite's foundations throughout ours planetary system as part or not of all the galaxies in our cosmos. The most significant finding and certainly surprising, published in this paper at first, refers to the significant implications of solar drag in planetary stability, which is conceptually very different from A. Einstein's deformation field's idea published in General Relativity (Albert Einstein, 1916). Another phenomenon also published at first time in this work and also very significant, is repulsive the electro-gravitational action force between planetary objects that deters and prevents possible collisions between them.

\subsubsection{Planetary Subspace - Physical Constants}

Values theoretically calculated on $\mathrm{TCS}_{\mathrm{PM}}$ for dynamic constant own of each heavenly body are:

$Y_{\mathrm{P}}$ for the specific case of the Sun's drag constant is $2.53265203011335 \times 10^{9} \mathrm{~s}^{-1} \cdot \mathrm{m}^{-1}$

$\varsigma_{3}$ the drag coefficient field correction for Earth is $2.2313002857048979 \times 10^{-4}$ dimensionless.

Each heavenly body according to its inertial mass and rotation speed has an eigenvalue of the planetary field drag of each star $\left(Y_{\mathrm{P}}\right)$ and drag coefficient field correction of each planet depending coordinates about its star $\left(\varsigma_{3}\right)$. The Sun's drag $\left(Y_{\mathrm{P}}\right)$ has an action's effect of the attraction on all existing bodies in the solar system.

The values theoretically calculated on TCS $_{\mathrm{PM}}$ for universal constants correlated with the inertial mass are:

$$
\begin{array}{ll}
\text { Electro-gravitational constant }\left(k_{G E}\right) & 2.88821919179004 \times 10^{-10} \mathrm{~N} \cdot \mathrm{m}^{2} \cdot \mathrm{kg}^{-2} \\
\text { Magneto-gravitational constant }\left(k_{G M}\right) & 3.57558690249434 \times 10^{-44} \mathrm{~N} \cdot \mathrm{s}^{2} \cdot \mathrm{kg}^{-2} \\
\text { Speed of gravitational waves }\left(c_{G}=\left\{k_{G E} / k_{G M}\right\}^{1 / 2}\right) & 8.98755178736818 \times 10^{16} \mathrm{~m} \cdot \mathrm{s}^{-1}
\end{array}
$$

The electro-gravitational force $k_{\mathrm{GE}}$ is equivalent to the Coulomb force, with a sense of repulsion between planetary objects that interact, in addition to this force is independent of the motion to hold the objects under consideration. The magneto-gravitational force $k_{\mathrm{GM}}$ is equivalent to the known Biot's Law in the atomic field.

\subsection{Galactic Subspace}

It is the subspace of third order and I have assigned this name because the galaxy stability of all the galaxy systems from the Cosmos depends on the galactic quarks. The galactic quarks are wave phenomena of this subspace. The galaxy stability is developed in the QGD theory "Quantum galactic-dynamics theory" that would be published soon.

All the phenomena described by the galactic-magnetism are within the subspace domain. These phenomena are determined by the interaction of galactic quarks that make up atomic particles that exert forces on other quarks of the same domain; the forces are transmitted by alteration and modification of the galactic subspace field that surrounds. All these phenomena of this subspace are not scientifically well known nowadays. Within the TCS theory can be considered dark matter or dark energy as the essence of the subspaces, because the elementary energy is manifested in four different magnitudes, is a fundamental attribute and it is owned by each of the four subspaces. In the TCS theory the energy is part of the essence of the four subspaces, and thus any 
entity natural or artificial that exists, occupies a portion of the field within each subspace that corresponds to a certain degree of quantized subespacial energy.

In TCS theory all natural or artificial entity that exists, even us, is just a cluster of complex wave phenomena organized, into a domain of one or more of the subspaces, and all are affected by the "devenir" determined by the subespacial temporary expansion.

\subsection{Temporal Subspace}

It is the subspace of fourth order, the latest and it is the larger subspace (enormously larger) that involves the full Universe. I have assigned this name because "the flow of time" (related to the Spanish word "devenir") is characteristic of them, given by the steady expansion of this subspace inside each Cosmos. The phenomena of this subspace are not scientifically well known nowadays, but the "absolute time" is included since several centuries on all physical laws of Dynamics.

\section{The Absolute Time}

The absolute time is equal to the absolute value of expansion of the temporary subspace happened between two instants, as you can see into the electromagnetic hysteresis on section 12.1. It is for this reason that the time always runs, although the object does move or does not move. In the event that you can make an instantaneous movement from one point to another into the temporary subspace, the time is zero, because there has been no expansion of the temporary subspace.

This new concept of the absolute time sets into physics and philosophy that although an object is not moved from a place, there is always a period of absolute time given by the "devenir" (or aging) given by the temporary expansion, question of the time that until now was not properly defined within the field of theoretical physics.

The "absolute time" or simply the variable " $t$ " in the TCS theory, is exactly equal to the absolute magnitude of the expansion of temporary subspace occurred between two specific moments. The variable "absolute time" is a scalar and is given by the following expression into the TCS theory:

$$
\left.t(\text { second })=k^{\prime} \text { (second }\right) \cdot V t_{2}\left(\mathrm{~m}^{3}\right) \cdot\left\{V t_{1}\left(\mathrm{~m}^{3}\right)\right\}^{-1},
$$

where $V_{t 1}$ is for any volume within the temporary subspace in the initial moment, $V_{t 2}$ is the size reached due to the expansion of temporary subspace time measured at the final instant and $k$ ' is a dimensional constant which depends on the time unit and the measuring method taken.

In the theory of relativity, the absolute time is related to a shift in the temporal dimension; this is why the absolute time at TCS theory is conceptually different deep inside with the absolute time of the theory of relativity. But, unless the conceptual difference deep inside in the calculation equations, which contain the time variable " $t$ ", developed within the classical physics and relativity theory it is right within the TCS theory, if in the process the "absolute time" do not use mathematical expressions derived from the Lorentz transformation (1887) and do not use expressions that include the absolute time associated with the temporary displacement.

\section{The Physical Space}

In any essence of the nature, the objects, our creations and everything to what our senses give a form, there are only ordered systems of wave phenomena of the three subspaces of smaller order -or physical space- that travel for the temporary subspace of last order without losing their integrity. Our Cosmos in expansion -the physical space-, is a spherical portion or place of constant volume -see 13 Cosmological implications- of the real space integrated totally by four subspaces -the Universe-, however, where in our Cosmos only resides a tiny fraction of the Universe or real space.

Due to it the coordinates of a point ( $p$ ) of the physical space or Cosmos are a function of homologous points in the three subspaces and then we can define it in Euclidean-Pythagorean form:

$$
p=\sqrt{\left(x_{\left(x_{a}, x_{p}, x_{\mathrm{g}}\right)}\right)^{2}+\left(y_{\left(y_{\mathrm{a}}, y_{\mathrm{p}}, y_{\mathrm{g}}\right)}\right)^{2}+\left(z_{\left(z_{\mathrm{a}}, z_{\mathrm{p}}, z_{\mathrm{g}}\right)}\right)^{2}},
$$

where $x_{\mathrm{a}}, y_{\mathrm{a}}$ and $z_{\mathrm{a}}$ there are the coordinates in the atomic subspace, $x_{\mathrm{p}}, y_{\mathrm{p}}$ and $z_{\mathrm{p}}$ there are the coordinates in the planetary subspace and $x_{\mathrm{g}}, y_{\mathrm{g}}$ and $z_{\mathrm{g}}$ there are the coordinates in the galactic subspace.

However, because all coordinated point of the matter always determines only a point into the atomic, planetary and galactic subspaces, to the one that we can name it like "integrity principle of the matter and cosmos", settled down by the relative immobility of these three subspaces, we can put simply, determined by the univocal correspondence among the points of the three subspaces -the physical space and metric space-: 


$$
\begin{aligned}
f_{\left(x_{\mathrm{a}}, x_{\mathrm{p}}, x_{\mathrm{g}}\right)}=f_{\left(y_{\mathrm{a}}, y_{\mathrm{p}}, y_{\mathrm{g}}\right)}=f_{\left(z_{\mathrm{a}}, z_{\mathrm{p}}, z_{\mathrm{g}}\right)} & \therefore \quad x_{\mathrm{a}}=x_{\mathrm{p}}=x_{\mathrm{g}}, \quad y_{\mathrm{a}}=y_{\mathrm{p}}=y_{\mathrm{g}} \quad \text { and } \quad z_{\mathrm{a}}=z_{\mathrm{p}}=z_{\mathrm{g}} \\
\text { then } \quad p & =\sqrt{\left(x_{\left(x_{\mathrm{a}}, x_{\mathrm{p}}, x_{\mathrm{g}}\right)}\right)^{2}+\left(y_{\left(y_{\mathrm{a}}, y_{\mathrm{p}}, y_{\mathrm{g}}\right)}\right)^{2}+\left(z_{\left(z_{\mathrm{a}}, z_{\mathrm{p}}, z_{\mathrm{g}}\right)}\right)^{2}}=\sqrt{x^{2}+y^{2}+z^{2}},
\end{aligned}
$$

this is why I have not included the temporary subspace in the calculation of the coordinates of a point.

\section{The Matter}

The matter it is all physical entity constituted by one or many atoms. The atoms are organized systems of atomic particles, all orbiting on a common center and into electromagnetic-dynamic equilibrium. The study of the atomic stability is developed in the QEDa theory. The elementary substances are integrated by one or more atoms of same structure and composition, all their atoms are of same inertial mass number "A" (is equal to the total number of protons which contains the atomic nucleus) and same atomic number " $Z$ " (is equal to the number of electrons which contains the atom in its non-ionized state). These particles that constitutes the atoms are only three, the electron, the proton and the negatron (the number of negatrons existing on atomic nucleus -in non-ionized state- is equal to " $\mathrm{A}$ " less " $\mathrm{Z}$ ") and all they are integrated by quarks.

\section{The Quarks}

The quarks are only wave phenomena and domain waves of each subspace; the study on the quarks is developed in the "Quantum electrodynamics wave theory" QEDw theory that would get published in the future. These are the elementary components of the atomic particles. Two only quarks exist inside each subspace, both with equal charge and different sign, one of negative charge (always of action effect of repulsion -cavitations waves-) and the other with positive charge (always of action effect of attraction -compression waves-), under of Coulomb and Gauss' laws.

\subsection{Electric Quarks}

In the atomic subspace, of first order, we have the electric negative quark, represented (because it has other quarks unknown today) by the elementary particle denominated as electron, and the electric positive quark, represented (ditto previous) by the elementary particle denominated called positron (it is originated by disintegrating the negatron or proton). Both quarks are perfectly well known nowadays.

\subsection{Planetary Quarks}

In the planetary subspace, of second order, we have theoretically the planetary negative quark and the planetary positive quark. These quarks are not known nowadays. The planetary quarks into the planetary subspace domain are a part of the atomic particles; the planetary quarks are equivalent to the gravitons of contemporary theories. The planetary quarks determine the stability of planetary systems. While in the galactic stability are involved the galactic quarks that are within the domain of the galactic subspace and also these quarks are into the atomic particles, and therefore belong to a different domain that the planetary quarks or the gravitons, the study falls completely within the QEDp theory and QEDw theory.

\subsection{Galactic Quarks}

In the galactic subspace, of third order, we have theoretically the galactic negative quark and the galactic positive quark. These quarks are not known nowadays.

Note: In theoretical form there are not the temporary quarks.

\section{The Atomic Particles}

Like I have already mentioned, the particles that exist in substances in the nature are only three; all of them consist of a certain quantity of quark characteristic of each subspace, this subject would be developed in the QEDw theory.

\subsection{Electron}

It is a particle with negative electric charged and positive planetary charge. Outside the atom it is absolutely stable due to the quarks that make up.

The inertial mass it is equal to the unit. The electron is composed with more than one type of quark, and with the current experimental technology it is only the detectable atomic quark with negative charge. Within the QEDw theory the concept of quarks is limited in term of charges; the quark are elementary units and therefore does not exist in the nature fractional charges, because the QEDw theory it's far from current conventional concepts. 
In QEDw theory all quarks interact among themselves only within their own domain, positive or negative the atomic quarks interact only with atomic quarks into the atomic subspace, negative or positive planetary quarks interact only with planetary quarks into the planetary subspace and negative or positive galactic quarks only interact with galactic quarks into the galactic subspace. There are also different magnitudes of absolute inertial masses and the charges within each domain, and also are different the magnitudes of the remaining physical constants within each domain.

In laboratory experiments with high energy particles, depending on the magnitude of the used energy, can are produced events related to atomic particles and quarks that does not exist in the natural substances, within these experimental phenomena is the fragmentation of the inertial masses and the fragmentation of the charges. I think that instead of expensive experiments with very high energies, we need experiments more original and subtle as the experiments performed by Dr. A. Aspect with electromagnetic waves, which will lead to better understanding the different types of waves that exist in nature. And then, the development of detectors for different types of waves, we must introduce in the experimental the study of quarks.

\subsection{Negatron}

It is a particle with negative electric charged and positive planetary charge. Outside the atom it is an unstable particle in normal condition due to quantity of the quarks that make up.

The inertial mass it is equal to three units. The properties of this particle were first published in the book QEDa theory and the neutron was eliminated in final form as nuclear particle.

The negatron is a part of the neutron and in any complex nucleus (two protons or more), with the exception of hydrogen 1 , is part of the inner layer of the nucleus (the protons are in the outer layer of the nucleus). The neutron is not a particle, it is an atom composed of one proton and one negatron that have been launched at high speed outside the nucleus, that remain in solidarity, due to the electrostatic interaction, and resulting in the disintegration of an atomic nucleus. The Neutron is a true atom, at rest can be detected by optical spectroscopy, within crystalline networks; this elemental substance I named it with the name of Cristyn in QEDa theory. The Cristyn or Neutron is composed of one proton and one negatron, the proton and negatron is mixed on the same plane, there are both particles in the form of a helix in the direction of advance of them, the proton turn in the cardinal radius orbit, while negatron tour in an orbit of greater radius. To the extent that increases the speed of the whole, reduce of the two radius helix and thus decreases the distance between the proton and negatron. To the extent that increases the speed, decrease of the helix radius, then we have: slow neutrons (of heavy frontal area, that are causing the disintegration of nuclei in nuclear fission), and fast neutrons and with very small helix front radius, that crossing the nucleus without interacting with them and are retained or disintegrated in the armor of the reactors. The neutrinos are the planetary quarks and the galactic quarks, to come off in the disintegration of an atomic nucleus or in the beta decay of neutrons or Cristyn. For the beta decay of neutron, the relations accurate inertial mass and charge of the quarks (atomic, planetary and galactic) are:

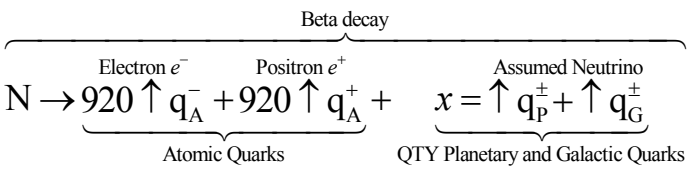

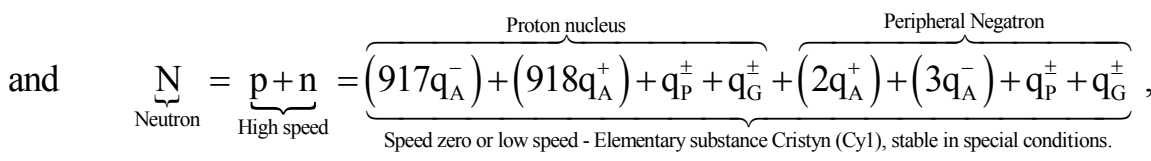

where " $\mathrm{N}$ " is a neutron, "p" is a proton, " $\mathrm{n}$ " is a negatron, " $\mathrm{x}$ " is the assumed neutrino or a number of planetary quarks and galactic quarks, $\mathrm{q}_{\mathrm{A}}{ }^{-}$is a atomic negative quark of inertial mass equal to the electron inertial mass, $\mathrm{q}_{\mathrm{A}}{ }^{+}$ is a atomic positive quark of inertial mass equal to the electron inertial mass, $\mathrm{qp}_{\mathrm{P}}^{ \pm}$are planetary quarks of charge positive and negative and $\mathrm{q}_{\mathrm{G}}{ }^{ \pm}$are galactic quarks of charge positive and negative, see note below.

The atomic quarks are detected with current laboratory techniques and are well known, while the remaining quarks are shown by their effect when they collide in a very sporadic due to its enormous speed.

The composition of galactic and planetary quarks and their inertial masses are very different from the composition of atomic quarks, the inertial mass release during beta decay of the non-atomic quarks, is that contemporary physics has been attributed to the alleged particle predicted in 1930 by Wolfgang Pauli and called neutrino. The planetary quarks and the galactic quarks, travel at enormous speeds greater than that the light, in 
some cases their effect is shown in laboratory experiments and has been attributed to the neutrino. Planetary quarks and galactic quarks released by the beta decay cannot interact with atomic quarks, but quarks can crash with quarks of the same domain, this is what happens when a quarks crash at great speed a quark of the electron and it is disintegrated releasing blue glow, like that seen in the Cherenkov radiation in the atmosphere and the interior of nuclear fission reactors.

Note: Not specified quantities of planetary and galactic quarks, because even, I have not completed the analysis of the QEDw theory.

\subsection{Proton}

It is a particle with positive electric charged, negative planetary charged and positive galactic charged, due to the quarks that make up what, and for the same reason outside the atom it is absolutely stable. In QEDa theory the inertial mass it is equal to one thousand eight hundred and thirty-five units.

\section{The Waves of Subspaces}

The wave radiation phenomena of each subspace travels within their own domain and also interacts with subspace of following order, what determines potential energy and kinetic cycles in the opposite way in both subspaces. This subject would get developed in the QEDw theory; we would be able to see a preview on Appendix A in section A.3. "Experimental Test of Bell's Inequalities Using Time- Varying Analyzers". The planetary waves, galactic and temporary travels at enormous speeds greater than the light it is not in conflict with the theory of relativity; in the theory of relativity are still unaware today the existence of subspaces and inside it says nothing of another kinds of electromagnetic waves, except in the few references made to gravitational waves. A. Einstein worked on general relativity even when D. Hilbert had not completed his work on the mathematical foundations of subspaces, but there was significant links between the two scientists that make me suspect that this item A. Einstein knew it, but most likely in a life is not enough to reach the final conclusions.

The speed of the wave of each subspace is characteristic and constant:

\subsection{Electromagnetic Waves}

These waves (electromagnetic radiation phenomena) are within the domain of atomic subspace and always traveling at a constant speed and equal to the well-known speed of light, equals $299,792,458 \mathrm{~m} \cdot \mathrm{s}^{-1}$. The representation of the full range of possible electromagnetic radiation waves, in this work it is called by the name of atomic spectrum -it is so called to distinguish it from other spectrums-.

\subsection{Gravitational Waves}

These waves (planetary-magnetic radiation phenomena) are within the domain of planetary subspace and always traveling at a constant speed and equal to the speed of light elevated to the second power, equals $8.98755179 \times 10^{16} \mathrm{~m} \cdot \mathrm{s}^{-1}$. The representation of the full range of possible planetary-magnetic radiation waves is called by the name of planetary spectrum.

\subsection{Galactic Waves}

These waves (galactic-magnetic radiation phenomena) are within the domain of galactic subspace and always traveling at a constant speed and equal to the speed of light elevated to the fourth power, equals $8.07760871 \times 10^{33} \mathrm{~m} \cdot \mathrm{s}^{-1}$. The representation of the full range of possible galactic-magnetic radiation waves is called by the name of galactic spectrum.

\subsection{Temporary Waves}

These waves (temporary-magnetic radiation phenomena) are within the domain of temporary subspace and always traveling at a constant speed and equal to the speed of light elevated to the eighth power, equals $6.52477625 \times 10^{67} \mathrm{~m} \cdot \mathrm{s}^{-1}$. The representation of the full range of possible temporary-magnetic radiation waves is called by the name of temporary spectrum.

\section{Scope of the Effects of Magnetic Field}

The magnetic field effects within each subspace are limited in scope, is not infinite as hitherto has been assumed. The scope is equal to the maximum speed of the sub-space divided by the absolute time unit (one second). For intense that can be the magnetic fields effects cannot exceed the following limits:

(a) Inside the atomic subspace for the electromagnetic phenomena the limit of the magnetic field effects is $2.99792458 \times 10^{8}$ meters. 
(b) Inside the planetary subspace for the gravitomagnetic phenomena the limit of the magnetic field effects is $8.98755179 \times 10^{16}$ meters.

(c) Inside of galactic subspace for the galacticmagnetic phenomena the limit of the magnetic field effects is $8.07760871 \times 10^{33}$ meters.

(d) Inside of temporary subspace there are no magnetic fields due to the lack of quarks.

\section{Example}

The TCS theory offers us a new and simple vision of our cosmos. To get a quick understanding of this theory take the following example:

If in a small container with lid, filled with water, blend well be a certain amount of cooking salt, equal amount of sugar and equal amount of pepper and then introduce it in a larger container filled with water.

The small container represents us the limits of our Cosmos. The water outside and inside us represents the temporary subspace.

If we extract the stage cooking salt phase, sugar phase and pepper phase and then introduce in three new containers of equal size of small container and relative position as the space they had, these containers we represent the three subspaces -atomic subspace, planetary subspace and galactic subspace.

Note that the elementary volumetric units of each component and the total volume of water in the container lower must necessarily be equal to the total volume of salt extracted, the total volume of sugar extracted and the total volume of pepper extracted, because if this does not happen there would be no physical continuity.

\section{The Redshift (Brief Historical Account of the Redshift)}

The observed redshift in the electromagnetic spectrum or atomic spectrum in distant stellar objects where absorption lines of known substances are visually displaced toward infrared this led to a profuseness of cosmological theories. Their discovery is due to Vesto Melvin Slipher (1875/1969) from Lowell Observatory on 1912, more lately this theory was confirmed by the American astronomers of Mount Wilson Observatory, Milton L. Humason (1891/1972) and Edwin Powell Hubble (1889/1953) who published it in 1928. This redshift phenomenon was attributed to the galaxies remoteness at a speed of recession that increases proportionately with the distance.

This redshift of the atomic spectrum was attributed to Doppler Effect (1842), and it is due to the length of the wavelength of each cycle, on directly proportional form to the speed of recession of the source that moves away from observer point.

Promptly was put to rest that every stellar object were moving away, from each other, at a rate given by a expansion constant (called it Hubble constant) and initially calculated by himself at $540 \mathrm{Km} \cdot \mathrm{s}^{-1} / \mathrm{Megaparsec}^{-1}$. As cosmological conclusion was extended an idea that everything was a result of a great initial explosion -the big bang-, which gave beginning to the whole universe or cosmos -the big-bang theory can be included into the TCS theory if the scale of cosmological time is extended-.

The inverse of the expansion constant is the time elapsed from the explosion time was happened to the present time, which gives us a life of $1.8 \times 10^{9}$ years, a value too small for the reality that surrounds us. Astronomers Walter Heinrich Wilhelm Baade (1893/1960) and Allan Rex Sandage (1926/2010), regardful to this pointless theory and in order to get closer to a more realistic scenario, they saw by put right the expansion constant were able to match the Big Bang Theory by Georges Henri Joseph Eduardo Lemaitre (1894/1966) with a more conservative, between 75 and $100 \mathrm{Km} . \mathrm{s}^{-1} / \mathrm{Megaparsec}^{-1}$ of the Hubble constant. The latest value determines a mean lifetime of $1.3 \times 10^{10}$ years, on the assumption that the speed of expansion on coursed time has remained constant, because if not the time to compute should be much lower. At present, the Hubble constant magnitude is estimated about $77 \mathrm{Km} \cdot \mathrm{s}^{-1} /$ Megaparsec $^{-1}$.

\section{The Electromagnetic Hysteresis}

The electromagnetic waves are moving within their domain, the atomic subspace. Any electromagnetic wave propagates in successive cycles, swinging in a continuous change, and if this medium should be elastically perfect and time is unchanged (there isn't electromagnetic hysteresis), the wave movement will continue forever unless something stands on their way. But, in nature there isn't an eternal phenomena of wave propagation, all wave will spread across sooner or later and disappear braking by their environment or diffused by it. The wave phenomenon braking is determined by the electromagnetic hysteresis of the medium, while the diffusion is determined by the unary energy of the subspace their domain. When the wave reaches a very little energy value, 
the momentum it becomes less than the value of unary energy of the subspace of their domain, and consequently is absorbed by the medium and disappears. The electromagnetic hysteresis (energy loss) is the reason why the free space impedance $\left(Z_{0}\right)$ is not equal to zero.

\subsection{Determination of the Constant Electromagnetic Hysteresis}

The total distance travelled by an electromagnetic wave is possible to be known because it is equal to the sum of all cycle wavelengths that elapsed from the journey of the source to the observer. The successive cycle wavelengths from the wave irradiation will be increasing proportionately to the energy loss in each of them.

This energy loss is directly proportional to the initial energy of the whole wave and always multiple Planks' constant by the quantum constraints.

The distances covered can be calculated in accordance with the following reasoning:

$$
\begin{array}{r}
d=\lambda_{1}+\lambda_{2}+\lambda_{3}+\ldots+\lambda_{n-1}+\lambda_{n}=\overbrace{\lambda_{0} k_{h}}^{\lambda_{1}}+\overbrace{\lambda_{0} k_{h} k_{h}}^{\lambda_{2}}+\overbrace{\lambda_{0} k_{h} k_{h} k_{h}}^{\lambda_{3}}+\ldots+\overbrace{\lambda_{0} k_{h}^{n-1}}^{\lambda_{n-1}}+\overbrace{\lambda_{0} k_{h}^{n}}^{\lambda_{n}} \\
=\lambda_{0} k_{h}+\lambda_{0} k_{h}^{2}+\lambda_{0} k_{h}^{3}+\ldots+\lambda_{0} k_{h}^{n-1}+\lambda_{0} k_{h}^{n}=\lambda_{0}\left(k_{h}+k_{h}^{2}+k_{h}^{3}+\ldots+k_{h}^{n-1}+k_{h}^{n}\right) \\
=\lambda_{0} \sum_{i=1}^{n} k_{h}^{i} \quad \text { then } \quad d=\lambda_{0} k_{h}\left(k_{h}^{n}-1\right)\left(k_{h}-1\right)^{-1},
\end{array}
$$

where $d$ is the distance between the emission source and the observer, $\lambda_{\mathrm{x}}$ is the wavelength of a given cycle, $\lambda_{0}$ is the initial or origin wavelength emitted and $k_{\mathrm{h}}$ is the constant of electromagnetic hysteresis. As previously explained on the cycle wave series, the wavelength of the last cycle upon arrival at the observer is equal to the product of the initial wavelength per the factor by electromagnetic hysteresis to the power of the number of cycles and then we can state:

$$
\lambda_{n}=\lambda_{f}=\lambda_{0} k_{h}^{n} \quad \therefore \quad k_{h}^{n}=\lambda_{f} \lambda_{0}^{-1}
$$

Finally, the distance traveled by the electromagnetic wave can be calculated as follows:

$$
d=\lambda_{0} k_{h}\left(k_{h}^{n}-1\right)\left(k_{h}-1\right)^{-1}=\lambda_{0} k_{h}\left[\left(\lambda_{f} \lambda_{0}^{-1}\right)-1\right]\left(k_{h}-1\right)^{-1} \quad \text { then } \quad d=k_{h}\left(\lambda_{f}-\lambda_{0}\right)\left(k_{h}-1\right)^{-1}
$$

where $\lambda_{\mathrm{f}}$ is the end wavelength of arrival to observer.

The energy loss from each wave is a function of the wavelength of the initial wave or wave emitted and furthermore, necessarily must be related to the Planck's constant, because it also necessarily must be equal or multiple of this constant, but can never be less than the basic unit of quantum energy.

But, because the space is expanding as are the time and by studying the limits of expressions I conclude that the magnitude of the constant of electromagnetic hysteresis is given by the following expression:

$$
\begin{aligned}
\lim _{\lambda_{0} \rightarrow 0}\left\{\left[k_{h}\left(\lambda_{f}-\lambda_{0}\right)\left(k_{h}-1\right)^{-1}\right]-d\right\} & =\left[k_{h} \lambda_{f}\left(k_{h}-1\right)^{-1}\right]-d \\
\therefore \quad \lambda_{f} & =d k_{h}^{-1}\left(k_{h}-1\right) \quad \text { if } k_{h}^{-1}\left(k_{h}-1\right)=2 h \text { then } k_{h}=(1-2 h)^{-1}
\end{aligned}
$$

where $h$ is the Planck's constant and the constant of electromagnetic hysteresis is equivalent to the energy lost by each wave cycle.

The cause of the hysteresis is due to the expansion of temporary subspace and the physical space that produces a delay time of each wave cycle and a lengthening of the wavelength; and is determined by the Planck's constant per twice (due to the simultaneous expansion of time and space) is due to the limitations of quantum mechanics, as always energy losses must be multiple of this constant. The value obtained in exact form to constant electromagnetic hysteresis $k_{\mathrm{h}}$ is:

$$
k_{h}=(1-2 h)^{-1}(\mathrm{~J} . \mathrm{s})=1.000000000000000000000000000000001325213792 \text { Dimensionless }
$$

The value obtained in exact form to corrective factor by electromagnetic hysteresis $\psi_{\mathrm{h}}$ is:

$$
\psi_{h}=(2 h)^{-1}(\mathrm{~J} . \mathrm{s})=7.54595225341572541108886026846208 \times 10^{32} \text { Dimensionless }
$$


The calculation of the distance to stellar objects using the redshift $z$ and corrective factor by electromagnetic hysteresis $\psi_{\mathrm{h}}$, in function on the wavelength of origin $\lambda_{0}$ is:

$$
d=k_{h}\left(\lambda_{f}-\lambda_{0}\right)\left(k_{h}-1\right)^{-1}, \quad \psi_{h}=k_{h}\left(k_{h}-1\right)^{-1} \quad \text { and } \quad \lambda_{f}=\lambda_{0}(1+z) \quad \text { then } \quad d=\psi_{h} z \lambda_{0}
$$

\subsection{Other Variables of the Electromagnetic Wave}

Beginning from previous expression and expression (8), can be calculated the end wavelength depending on the distance, the distance from the observer source, the initial wavelength and the hysteresis of the atomic subspace, with the following expression:

$$
\lambda_{f}=\left[d\left(k_{h}-1\right)+k_{h} \lambda_{0}\right] k_{h}^{-1}=\lambda_{0}(1+z)
$$

Also, starting from the previous expression and end wave energy of the arrival to the observer can be calculated energy received with the following expression:

$$
\begin{aligned}
E_{f}=\operatorname{ch} \lambda_{f}^{-1}=\operatorname{ch}\left\{\left[d\left(k_{h}-1\right)+k_{h} \lambda_{0}\right] k_{h}^{-1}\right\}^{-1}= & \\
& \quad \operatorname{ch} k_{h}\left[d\left(k_{h}-1\right)+k_{h} \lambda_{0}\right]^{-1}=\frac{\operatorname{ch}}{\lambda_{0}} \frac{\psi_{h}-1}{\psi_{h}(1+z)}=\operatorname{ch}\left(\lambda_{0}(1+z)\right)^{-1}
\end{aligned}
$$

The quantity of wave cycles $n$ which occurred during the travel time of the wave, since their irradiation at source until they reach the observer, solved in the expression (8), is:

$$
n=\log \left(\lambda_{f} \lambda_{0}^{-1}\right)\left[\log \left(k_{h}\right)\right]^{-1}=\log (1+z)\left[\log \left(k_{h}\right)\right]^{-1}
$$

According to the redshift, and also, according to the maximum value of energy for any electromagnetic wave, the loss of time per unit due to the temporary expansion $P_{\mathrm{U}}$ in $\mathrm{s} . \mathrm{s}^{-1}$ necessarily equals the absolute value of the expansion in $\mathrm{m} . \mathrm{s}^{-1}$ of atomic subspace, planetary subspace and galactic subspace; is:

$$
\begin{aligned}
& P_{t}=\left(\lambda_{f}-\lambda_{0}\right)(2 c)^{-1} \quad T=c^{-1} k_{h}\left(\lambda_{f}-\lambda_{0}\right)\left(k_{h}-1\right)^{-1} \\
& \text { then } P_{U}=P_{t} T^{-1}=\left(\lambda_{f}-\lambda_{0}\right)(2 c)^{-1}\left[c^{-1} k_{h}\left(\lambda_{f}-\lambda_{0}\right)\left(k_{h}-1\right)^{-1}\right]^{-1} \\
& =\left(k_{h}-1\right)\left(2 k_{h}\right)^{-1}=\left(2 \psi_{h}\right)^{-1}=|h|=6.62606896 \times 10^{-34} \mathrm{s.s}{ }^{-1},
\end{aligned}
$$

where $P_{\mathrm{t}}$ is the lost time due to the expansion of the temporary sub-space of our cosmos and $T$ is the travel time of electromagnetic wave. The factor two in the above expression comes from the expansion of temporary subspace and atomic subspace. Then, the maximum value of energy $E_{M}$ for all electromagnetic wave is:

$$
E_{M}=f_{M} h=P_{U}^{-1} h=\left(6.62606896 \times 10^{-34}\right)^{-1} 6.62606896 \times 10^{-34}=1 . \text { Joule, }
$$

where $f_{M}$ is maximum value of frequency for any electromagnetic wave. The $E_{M}$ calculation is theoretical and comes from the theoretical relationship between the expansion of temporary subspace and physical space from of the equation (16). Then, the minimum value of wavelength $\lambda_{m}$ for maximum energy of the wave is:

$$
\lambda_{m}=f_{M}^{-1} c=6.62606896 \times 10^{-34} \times 299792458 .=1.9864455003959039 \times 10^{-25} \mathrm{~m} .
$$

\subsection{Calculating the Distance to Galaxies According to the Hysteresis}

I have calculated in the next section distances to three distant galaxies with the expression (12) -the Doppler Effect is not included on calculations- and then I compare them with astronomical distances estimated today through optics system. The values of the spectrographic series are exact and are extracted from QEDa theory and I have calculated all based on Lyman Alpha and Balmer Alfa, if the difference on the distances is very large. The total average error in the following calculations of distance respect to the optical observation is:

$$
n^{-1} \sum_{i=1}^{n} d d_{0 i}^{-1}=0,886123 \quad 11 \% \text { closer }
$$




\subsubsection{N.G.C. 2681 Ursa Major Galaxy}

The value of redshift $z=0.002455$ for hydrogen 1 estimated for this galaxy in NAS / IPAC Extragalactic database (1992).

$$
\begin{array}{r}
d=\psi_{h} z \lambda_{0}=7.54595225341572541108886026846208 \times 10^{32} \times 0.002455 \times 1.2156731231 \times 10^{-7} \\
=2.25207248462631 \times 10^{23} \text { meter } \\
d d_{0}^{-1}=2.25207248462631 \times 10^{23}\left(9.44841 \times 10^{23}\right)^{-1}=0.238355 \text { closer } 76 \%
\end{array}
$$

If calculated with Balmer Alpha $2^{S} \leftarrow 3^{S} \quad \lambda=6.5646348649 \times 10^{-7} \mathrm{~m}$.

$$
\begin{gathered}
\text { is } \quad d=1.21611914172785 \times 10^{24} \text { meter } \\
d d_{0}^{-1}=1.21611914172785 \times 10^{24}\left(9.44841 \times 10^{23}\right)^{-1}=1.28712 \quad \text { further } 29 \%
\end{gathered}
$$

where $d_{0}$ is the estimated distance in optical form.

12.3.2 N.G.C. 3115 Spindle Galaxy

The value of redshift $z=0.002235$ for hydrogen 1 estimated for this galaxy in NAS / IPAC Extragalactic database (1999).

$$
\begin{array}{r}
d=7.54595225341572541108886026846208 \times 10^{32} \times 0.002235 \times 1.2156731231 \times 10^{-7} \\
=2.05025743508750 \times 10^{23} \text { meter } \\
d d_{0}^{-1}=2.05025743508750 \times 10^{23}\left(9.43298 \times 10^{23}\right)^{-1}=0.21735 \text { closer } 78 \%
\end{array}
$$

If calculated with Balmer Alpha $2^{S} \leftarrow 3^{S} \quad \lambda=6.5646348649 \times 10^{-7} \mathrm{~m}$.

$$
\begin{gathered}
\text { is } \quad d=1.10713901497423 \times 10^{24} \text { meter } \\
d d_{0}^{-1}=1.10713901497423 \times 10^{24}\left(9.44841 \times 10^{23}\right)^{-1}=1.17369 \quad \text { further } 17 \%
\end{gathered}
$$

12.3.3 N.G.C. 4594-M104 Sombrero Galaxy

The value of redshift $z=0.003416$ for hydrogen 1 estimated for this galaxy in NAS / IPAC Extragalactic database (1992).

$$
\begin{gathered}
d=7.54595225341572541108886026846208 \times 10^{32} \times 0.003416 \times 1.2156731231 \times 10^{-7} \\
=3.13363731465723 \times 10^{23} \text { meter } \\
d d_{0}^{-1}=3.13363731465723 \times 10^{23}\left(2.77199 \times 10^{23}\right)^{-1}=1.13046 \text { further } 13 \%
\end{gathered}
$$

12.4 Calculating the Distance to Quasar According to the Hysteresis

I have calculated in the next section distances to three distant quasars, all based on Lyman alpha. Related to this topic see also section 12.2.

\subsubsection{Quasar APM 08279+5255}

The value of measured redshift $z=3.9122$ for hydrogen 1 estimated for this quasar in NAS / IPAC Extragalactic database.

12.4.2 Quasar CFHQS J2329-0301

$$
\begin{array}{r}
d=7.54595225341572541108886026846208 \times 10^{32} \times 3.9122 \times 1.2156731231 \times 10^{-7} \\
=3.58882198548068 \times 10^{26} \text { meter }=3.79338783181895 \times 10^{10} \text { light-years }
\end{array}
$$

The value of measured redshift $z=6.43$ for hydrogen 1 estimated for this quasar [27].

$$
\begin{aligned}
& d=7.54595225341572541108886026846208 \times 10^{32} \times 6.43 \times 1.2156731231 \times 10^{-7} \\
&= 4.97686232242895 \times 10^{26} \text { meter }=5.26054762563309 \times 10^{10} \text { light-years }
\end{aligned}
$$




\subsubsection{Quasar 3C273}

$$
\begin{array}{r}
d=7.54595225341572541108886026846208 \times 10^{32} \times 0.158339 \times 1.2156731231 \times 10^{-7} \\
=1.45250877858756 \times 10^{25} \text { meter }=1.53530299039513 \times 10^{9} \text { light-years }
\end{array}
$$

\subsection{Calculating the Wave Cycle}

The quantity of the wave cycle calculated with the expression (15), elapsed during the journey since the wave emission at source in NGC 2681 see section 12.3.1 until the observer on Earth is $1.85026101098116 \times 10^{30}$ wave cycles.

The Figure No. 1 shows the curves representing twelve electromagnetic waves within a range of typical energies covering the entire atomic spectrum.

Calculations are made by the expression (8), the constant of hysteresis of the atomic subspace given by the expression (9), the energy of coordinate axis " $y$ " and the distances of coordinate axis " $x$ ".

This figure clearly shows how is decreased the energy of all electromagnetic waves depending on the distance.

As long as grows the emission wave energy, decreases faster the energy that depended on the distance and hysteresis. All electromagnetic waves reach a point of extremely low energy.

Could be seen in the figure that initially the energy of electromagnetic waves decreases slowly also can reach a break point of the curve and from this point the energy of all the electromagnetic waves decreases maintaining exactly the same gradient. Also, you can notice that there is a maximum limit of energy emission of electromagnetic waves where energy decreases steadily with the already mentioned gradient.

Within the visible spectrum, for all emission of electromagnetic waves equal to or greater magnitude energy, due to the decrease of energy, the maximum distance that can be observed a radiant stellar object never can exceed $2.8 \times 10^{26}$ meters, equivalent to approximately $2.9 \times 10^{10}$ light years.

The minimum energy limit of every electromagnetic wave can be calculated based on condition that energy can never be less than the energy provided by the Planck's constant.

Starting from the expression (13) the energy minimum limit of all electromagnetic wave $E_{\mathrm{m}}$ is necessarily equal to the Planck's constant, then:

$$
E_{m}=\operatorname{ch} \lambda_{M}^{-1}=h \quad \therefore \quad \lambda_{M}=|c|=299792458 . \mathrm{m} . \quad \text { and } \quad f_{m}=c|c|^{-1}=1 . \mathrm{s}^{-1}
$$

where $\lambda_{\mathrm{M}}$ is the maximum wavelength and $f_{\mathrm{m}}$ is the minimum frequency. The maximum range of all electromagnetic wave $d_{\mathrm{M}}$ is:

\subsection{Calculating the Wavelength}

$$
\begin{array}{r}
d_{M}=7.54595225341572541108886026846208 \times 10^{32} \cdot\left(299792458 .-1.98645 \times 10^{-25}\right) \\
=2.26221957400214 \times 10^{41} \mathrm{~m} .=2.39116797646707 \times 10^{25} \text { light-years }
\end{array}
$$

The distance depends on the initial energy wave emitted and it is obvious that the wavelength of the initial wave or of origin cannot exceed the numerical value of the speed of light; in the case of electromagnetic waves within the spectrum that is being displayed in the figures above, the maximum distance cannot exceed $2.26 \times 10^{41}$ meters, equivalent to approximately $2.39 \times 10^{25}$ light years.

The Figure No. 2 is representative for electromagnetic wavelengths from the figure above. You can see clearly how to grow up wavelengths depending on the traveled distance by electromagnetic wave. In this figure can also be noticed a remarkable breakpoint in the growth up of wavelengths at which the entire electromagnetic wave takes on a gradient with growth up of the constant magnitude.

The wavelengths for very low energies are extremely long to reach the limit given by Planck's constant. These figures show without the slightest doubt that the observations made by instruments sensitive to very low energy, as the case by radio telescopes can greatly exceed to optical instruments and being able to overcome these instruments, because the wavelength that can be observed, $1 \times 10^{34}$ meters, distances are higher in $100,000,000$ times greater than the scope of any optical instrument. 


\subsection{Energy Radiated by the Sun}

The Earth receives from the sun a wide range of electromagnetic waves, but the energy of them suffers a fall due to an electromagnetic hysteresis and a wide range of electromagnetic waves, but the energy of them suffers a fall due to an electromagnetic hysteresis.

Due to the distance that we separate us from the Sun and the hysteresis of atomic subspace we don't receive electromagnetic gamma waves at very high frequencies that were emitted by the Sun. We have still not made experiments of energy radiated by the Sun in its vicinity. The calculations in the figure No. 3 were made on the assumption that the energy in the process of nuclear fusion in the Sun generates electromagnetic waves at the maximum energy and then, due to the distance traveled, their energy decays determined by the electromagnetic hysteresis.

The electromagnetic waves of very high energy during the travel lose much of their energy before reaching Earth. The Figure No. 3 shows that the maximum energy of electromagnetic waves that receives the Earth cannot exceed $2 \times 10^{-3}$ Joule.

In the case of Venus and especially Mercury, the received spectrum of high energy is very different than the received on Earth.

\section{Stellar and Cosmological Phenomena}

The phenomena stellar and cosmological observed currently are:

\subsection{Novas}

The nova it is a cataclysmic of a sun nuclear explosion type that depending on their magnitude could cause the breaking of symmetry of the atomic sub-space on their environment. The mean lifetime of the suns is approximately $1 \times 10^{10}$ years.

\subsubsection{Pulsar}

When the cataclysm of a massive Sun -nova- determines the breaking of symmetry of the atomic subspace there is originated what today is known as Pulsar. The periphery area of the breaking of symmetry acts as a mirror, reflecting the electromagnetic waves inwards and producing gamma-ray bursts of very high energy. If the sun is very massive, the area involved in the atomic subspace where is started the breaking of symmetry remains in successive cyclical movements like a shutter release in a cyclical shape of electromagnetic radiation.

\subsection{Supernovas (Quasar)}

The supernova is a cataclysmic of a galaxy nuclear explosion type that depending on their magnitude could cause the breaking of symmetry of the atomic and planetary sub-spaces. Usually the supernova originates one or more pulsars. The mean lifetime of the galaxies is approximately $1 \times 10^{18}$ years.

\subsection{Macronovas}

The macronova is a cataclysmic of a cosmic nuclear explosion type that causes the breaking of symmetry of the atomic, planetary and galactic sub-spaces. The mean lifetime of the Cosmos is approximately $1 \times 10^{36}$ years.

\section{Cosmological Implications}

\subsection{Implications on the Universe}

The Universe is completely submerged into the temporary subspace, it's simply everything that exists and it is populated by countless cosmos of equal size. The cosmos is a tiny portion almost spherical of the Universe. The cosmos are like spherical clouds isolated from each other, only the spectrum irradiated should vary depending on the state and age. To understand their properties and details, it is necessary to develop technology derived from the galactic-magnetic radiation phenomena -galactic spectrum- and temporary-magnetic radiation phenomena -temporary spectrum- due to their outstanding range in distance, $6.1 \times 10^{66}$ meter, around $6.4 \times 10^{50}$ light-years and $4.9 \times 10^{100}$ meter, approximately $5.2 \times 10^{84}$ light-years respectively.

All spaces of the inter-cosmos -the real space (all the subspace) around all cosmos-, remains in a perfect equilibrium of energy and density as well, all its volume expands due to the wind subspace received from the different cosmos due to the cubic expansion of its internal subspaces involved.

We can imagine the picture of the universe as a dark sky illuminated by innumerable cosmos, as if there were stars. Sometimes some of those points of light reach a remarkable luminosity, which then vanish into the dark sky due to the cataclysm of a cosmos -the macronova-, that in the future after many millions of years, will be regenerated again. 


\subsection{Implications on the Our Cosmos}

The Cosmos are formed by countless galaxies. Galaxies within the cosmos are in constant motion; it's very similar to the Brownian motion of molecules inside a gas and the oldest galaxies are located the nearest center of the cosmos. The physical space (subspaces atomic, planetary and galactic) and the temporary subspace in our cosmos are in continuous and constant expansion at a value of $6.62606896 \times 10^{-34} \mathrm{~m} \cdot \mathrm{m}^{-1}$-to physical space- or $6.62606896 \times 10^{-34} \mathrm{~s} . \mathrm{s}^{-1}$-to temporary subspace- .

The redshift phenomenon on the atomic spectrum of substances known today is in fact unequivocally due to a the electromagnetic hysteresis by expansion of the physical and temporary subspace, this cubic expansion of subspaces is not involved at all in the movement that is observed in the stellar objects, the movements observed are due to interactions with each other objects and not to the cubic expansion.

The travel of distant stellar objects are made at very low speeds from a few hundreds to a few thousands of kilometers per second and taking into account the stellar distances the Doppler Effect is a minimal influence on observed atomic spectrum.

The radius of our Cosmos is approximately $2.3 \times 10^{41}$ meters or $2.5 \times 10^{25}$ light-years -vastly larger than the current astronomical estimation-, this size has remained constant since its origin, only slowly changing its density of all subspace in our cosmos determined by the cubic expansion. At the periphery of our cosmos due to expansion, the wind speed of subspace exceeds the speed of light, as a result, our cosmos is surrounded by an unstable layer peripheral of internal radius of $2.26 \times 10^{41}$ meters, approximately $2.39 \times 10^{25}$ light years -the unstable layer peripheral of the cosmos has extremely low thickness- where due to the violent turbulence, pressure waves and cavitations are generated making the origin of quarks and then after a reordering of elementary particles and finally, the atomic hydrogen. In the TCS theory the radius of our Cosmos is huge, much larger than the size assumed by current theory, it is also much larger than the area observed by astronomical means. Our Cosmos is just a small part of the Universe occupied in full by the temporary subspace or more simply, is all that exists. Our Cosmos necessarily correspond to the maximum range of an electromagnetic wave, because at that distance or radius of the Cosmos, is the periphery where the wind produced by the expansion subespacial reaches the speed of light, is the limit of all Cosmos or also it is the place where the unstable outer layer of Cosmos. All Cosmos are the same size and only differ in the age and interior structure.

The make a relationship among String and Black Holes theories with the TCS theory. A cosmological event of quarks, elementary particles and substances generation within the peripheral layer of Cosmos, due to the existence of a cubic expansion that was established after the initial central singularity of each Cosmos. This cyclic cosmological event results in a macronova (big implosion) and creates a black hole, repeated $\mathrm{n}$ times into the Universe. This theory shares at several points within the String theory and Black Holes theory developed by Sir Stephen W. Hawking. To unify these two theories with the TCS theory is necessary to make minor changes on them to match the conceptual aspects about the origin (due to a initial singularity), the mechanism of cosmological evolution (a cubic centrifugal expansion of the subspace and material regeneration of the physical space on the most outer layer of Cosmos in centripetal form) and temporal and dimensional aspects (a physical space of nine dimensions and twelve dimensions in total, comprising the three-dimensional subspace plus the absolute time determined by the magnitude of expansion).

Our galaxy (the Milky Way) is located near its periphery of the cosmos, some $1.58 \times 10^{32}$ meter approximately $1.67 \times 10^{16}$ light-years and far from its center, calculation based on the redshift of the band of 21 centimeters and the distribution of energy received by the Earth from the heavenly sphere.

Within of the matter, the atomic systems -the atoms- interacts with each other due to electrical and magnetic atomics interactions - of the atomic subspace- making the determination of the outstanding stability of every atomic system. Also, within a galaxy and in their nearby, within the globular clusters, the planetary systems interacts with each other due to electric and magnetic planetary interaction -of the planetary subspace- avoiding the collisions and making a determination of the movements within the galaxy or globular cluster. Inside the cosmos and between the galaxies, happens the same among the planetary systems and in this case, is due to an electric and magnetic galactic interaction-of the galactic subspace-. This link of the systems at three levels makes the cubic expansion of subspaces has a minimal influence on the movements of stellar objects.

\subsection{Sequence of Cosmological Events}

The sequence of events that occurred at a general level from the birth of our cosmos is: 
- About $1 \times 10^{30}$ years ago, slowly began the cubic expansion of the four subspaces within our cosmos in all its volume, approximately $2.3 \times 10^{41}$ meters of radius -slightly less than the range of electromagnetic waves-.

- This expansion of subspaces turned out in the displacement of the peripheral of atomic subspace at enormous speeds that determined a turbulent flow and density variations at the periphery of the cosmos. As a result atomic, planetary and galactic waves were generated with very high intensity, which ended in the birth of the firsts quarks.

- Once the density of quarks, reached a high level throughout the unstable layer peripheral, began its reorganization -involving the other subspaces by interaction-in more complex systems, the atomic particles -only the electrons and protons-, which then also began to reorganize into more complex forms giving rise to atomic hydrogen within the unstable layer peripheral.

- This large cloud of atomic hydrogen that filled almost the whole of our cosmos, due to atomic electric interactions migrates below the unstable layer peripheral, and disintegrated into smaller clouds and formed the first galaxies. This is where born the first galactic cataclysms -supernovas- by gravitational attractions, also novas and pulsars, begin to form the first heavier elements than hydrogen. Within our cosmos all galaxies are in constant and continuous reorganization due to the increased amount of them.

\section{Conclusion}

Our current reality allows us to see into the past and by logical consequence in the future that in our planetary systems and Earth. It is also a reality, the existence in the past of glaciations periods that no surprise occur in the future, too our sun has a life period, after which it will occur a nova vanishing all signs of life in our planetary system, as happened many times. As a result, we should feel obliged to achieve practical utilization of nuclear fusion and the interplanetary navigation using the planetary field effect.

The four subspaces -atomic, planetary, galactic and temporary- have the property of absolute continuity, independently of one another, but also the subspaces can be considered a discrete shape, while the unit volume $-4 \pi \mathrm{k}^{\prime} \mathrm{h}^{3} / 3$ - of the Planck spherical constant being for the atomic subspace equal to $1.21858678364108 \times 10^{-99} \mathrm{~m}^{3}$; $\mathrm{k}$ ' is a constant specific to each subspace given by natural order number.

This theory allows a new interpretation of already made and remarkable experiments into the laboratories. In the Appendix A is mentioned the Michelson and Morley experiment (1887) that proves the field that integrates and surrounds us belongs to atomic subspace, turn round and it is in harmony with Earth. Also, it is explained the experiment of Dr. A. Aspect (1982), which for the first time is detected another kind of non-electromagnetic wave that travels exceeding the light speed, as you can see in section 8.2.

Beginning of experiment of Dr. A. Aspect using planetary waves, we can start working on the development of the RADARs and systems of communications much more efficient than the current ones, because they have speeds exactly one hundred times faster than electromagnetic waves. This technology would enable us to make it automatically an early warning of asteroids that may endanger the Earth and their destruction by means like LASER of the planetary waves or galactic waves. In the future can be used the same principles to develop and use galactic waves and temporary waves, as you can see sections 8.2 and 8.3 .

\section{Acknowledgments}

The most sincere gratitude to Professor Emeritus of KTH (Stockholm, Sweden) Dr. Bo Lehnert by his smart and clear questionnaire, that allowed better understanding of this work in the first edition (2010).

\section{References}

Alan, P. Lightman, \& Roberta, Brawer. (1990). Origins: the lives and worlds of modern cosmologists. Harvard University Press.

Ampère, André-Marie. (2000). Manuscrits sont valorisés par le Centre de recherche en histoire des sciences et des technique. (Unité mixte CNRS/Cité des sciences et de l'industrie).

Aspect, Alan J. Dalibard, \& G. Roger. (1982). Experimental Test of Bell's Inequalities Using Time-Varying Analyzers. Physical Review Letters, 49(25), 1804-1807. http://dx.doi.org/10.1103/PhysRevLett.49.1804

Baade, Wilhelm Heinrich. (1970). Walter reference: Sally H. Dieke, Baade, Wilhelm Heinrich Walter, Dictionary of Scientific Biography 1. New York: Charles Scribner's Sons. 352-354.

Baggley, Glenn, David, Burstein, Matthew, Colless, Roger, L. Davies, Robert, K., McMahan Jr, R. P., Saglia \& Gary, Wegner. (1999). Royal Astronomical Society, 1999 MNRAS.305-259W. 
Biot, Jean-Baptiste, \& Savart, Félix David Jeffrey Griffiths. (1999). Introduction to Electrodynamics( $3^{\text {rd }}$ Ed.). London, Prentice-Hall International Limited.

Bottinelli, L., Durand, N., Fouque, P., Gouguenheim, L., \& Paturel, G. (1992). Catalogue of optical radial velocitie. Observatoires de Lyon et Paris-Meudon, 1, p.1.

Butterworth-Heinemann. (1980). L. D. Landau, E. M. Lifshitz, pp.1-402.

Caminoa Lizarralde Daniel E. (2005). Teoría QEDa - El átomo y su núcleo, (in Spanish). October 25, Argentine 2005, 1-418. http://danielcaminoa.com.ar

Caminoa Lizarralde Daniel E. (2007). The helium family - Stability and gyromagnetic ratios. May 10, Argentine 2007, 1-80.

Caminoa Lizarralde Daniel E. (2007). The hydrogen family - Stability and gyromagnetic ratios. February 6, Argentine 2007, 1-58.

Caminoa Lizarralde Daniel E. (2008). Speed of Atomic Particles and Physical Constants. January 31, Argentine 2008, pp 1-16, second edition February 28, Argentine, 1-18. Adv. Studies Theor. Phys., 3(5), 221-237, Hikari Ltd.

Caminoa Lizarralde Daniel E. (2010). The Three-dimensional Complex Space theory, TCS theory. Advanced Studies in Theoretical Physics, 4(7), 323-351, Hikari Ltd.

Caminoa Lizarralde Daniel E. (2011). TCS Theory - Planetary Mechanics. Applied Physics Research, 3(2), November 2011. http://dx.doi.org/10.5539/apr.v3n2p89

Coulomb, Charles-Augustin. (1971). Physics and Engineering in Eighteenth Century France (Princeton, N. J., 1971, C. S. Gillmor).

Einstein, Albert. (1915 \& 1916). Die Feldgleichungen der Gravitation \& Die Grundlage der allgemeinen Relativitätstheorie. Berlin (1915 and 1916).

Einstein, Albert. (1986). Einstein's Legacy: The Unity of Space and Time. Scientific American Library, Nueva York, Schwinger, 1-250.

Euclid, Benno Artmann. (1999). The Creation of Mathematics. New York: Springer-Verlag.

Faraday, Michael. (2004). Experimental Researches in Electricity, (Three volumes), Julio 15, 2004. L. Pearce Williams. (1965). Michael Faraday, A. Biography. New York: Basic Books, 1965. http://www.amazon.com/

Gauss, Johann Carl Friedrich. (1981). Gauss A Biographical Study. Berlin - Springer-Verlag, Walter K. Bühler, $1-208$.

Hertz, Heinrich Rudolf. (2006). Untersuchungen über die Ausbreitung der elektrischen Kraft (VDM Verlag, $1-368$.

Hilbert, David. (1899). Grundlagen der Geometrie. Retrieved from http://www.amazon.com/

Hubble, Edwin Powell. (1929). A Relation between Distance and Radial Velocity among Extra-Galactic Nebulae. Proceedings of the National Academy of Sciences of the United States of America, Volume 15, Issue 3, 168-173. http://dx.doi.org/10.1073/pnas.15.3.168

Huygens, Christiaan. (1935). "Le Séjour de Christian Huygens à Paris et ses relations avec les milieux scientifiques français" (H. L. Brugmans).

Krasnosel'skii, M. A., \& Pokrovskii, A. V. (1989). Systems with Hysteresis. Berlin-Heidelberg-New York-Paris-Tokio: Springer Verlag, 1989, pp410.

Lemaître, Georges Henri Joseph Éduard. (1927). Un Univers homogène de masse constante et de rayon croissant rendant compte de la vitesse radiale des nébuleuses extragalactiques. Annals of the Scientific Society of Brussels 47A: 41.

Lorentz, Hendrik Antoon. (1892). La théorie électromagnétique de Maxwell et son application aux corps mouvants. Archives néerlandaises des Sciences exactes er naturelles. Netherlands.

Maxwell, James Clerk. (1994). The Classical Theory of Fields.

Michelson, Albert A., \& Morley, Edward W. (1887). On the relative motion of the Earth and the aluminiferous Aether. Philos. Mag. S.5, 24(151), 449-463. 
Newton, Sir Isaac. (1686). Philosophice Naturalis Principia Mathematica. July 5.

Olivier, Darrigol. (2003). Electrodynamics from Ampere to Einstein. Oxford University Press.

Peano, Giuseppe. (1889). Arithmetices principia, nova methodo exposita. Retrieved from http://www.amazon.com/

Peter M. Schuster. (2005). Moving the Stars - Christian Doppler: His Life, His Works and Principle, and the World After. Pöllauberg, Austria: Living Edition.

Poynting, John Henry Canadian Libraries. (1920). Internet Archive, Collected scientific papers Retrieved from http://www.archive.org/

Quasar Survey. (2007). Four Quasars above redshift 6 discovered by The Canada-France High-Z. ArXiv:0706.0914v2 [astro-ph] 30 Aug 2007.

Slipher, Vesto Melvin. (1912). The radial velocity of the Andromeda Nebula. Lowell Observatory Bulletin, pp.2.56-2.57, September 17 .

\section{Appendix A}

There have been scientific experiments whose interpretation is wanted to review.

\section{A.1 On the Relative Motion of the Earth and the Aluminiferous Ether}

Through an advanced instrument by the time, an optical interferometer developed by Albert A. Michelson, Michelson and Morley tried to show in 1887 the movement of the Earth meanwhile it moves through "Aether", under the hypothesis then, the substance that was the universal medium of electromagnetic waves and matter.

To everyone's surprise at that time and without finding any explanation, they did not detect any difference in the speed of light, regardless of the direction in which the measurement was made, in terms of spatial position of the Earth and their movements. Despite the enormous precision achieved today with this kind of instruments, has not yet found any variation on the speed of light to show evidence of some movement of the Earth, detected perfectly today through of gyroscopes or simple gravity pendulum. What is happening?

\section{A.2 Interpretation within TCS Theory of Constant Speed of Light on Earth}

In fact the key is obviously that experimentally proved that at the Earth's surface, there is an absolute stability of the speed of light, independently of the direction taken, while the instrument is jointly binding to surface.

This experiment demonstrates that the electromagnetic field or portion of atomic subspace surrounding the globe and that integrates the Earth, traveling together and in jointly binding form in orbit around the Sun. And as the visible spectrum and all electromagnetic wave moves in atomic subspace which is their domain at the speed of light, every electromagnetic wave entering this field, acquires the speed of light, with respect to the coordinates jointly binding to the earth and not with respect to stellar space that surrounds, and hence it does not show any variations.

This will occur the measure was made jointly binding form and at sea level or at peaks with Earth, commonly within our atmosphere, since the electromagnetic field in movement with Earth only has some hundreds of kilometers over atmosphere and away from it exist an unstable atomic subspace field, corresponding to the area of breakage of the symmetry atomic subspace see Figure No. 4.

The locking or jointly binding of the atomic subspace with the Earth is due to the action of neutralizing the effect of the hysteresis of atomic subspace as a result of the movement of particles in orbit of all atoms that make up the globe and their atmosphere. If you install an optical interferometer in a satellite into a geostationary orbit over the area of fracture of the symmetry atomic subspace, can be measured with absolute precision differences in speed of light due to the Doppler Effect, according to the coordinate axis that are taken, besides to direction and intensity of sub-spatial wind which is equal to 0.999 of the light speed in our galaxy due to the proximity of the unstable peripheral layer.

\section{A.2.1 Interpretation within TCS Theory of Coriolis Effect}

The Coriolis Effect -in honor of Gaspard-Gustave de Coriolis who discovered it in 1835- is also due to the phenomenon locking or jointly binding of atomic subspace field with Earth. The Eötvös Effect -in honor of Baron Roland von Eötvös- occurs for the same reason that the Coriolis Effect. Their explanation is very simple and is due to the motion of the atomic subspace field in conjunction with Earth, regarding to relative immobility 
of the subspaces, planetary and galactic -the wind speed of subspace has no effect on this phenomenon-. See Figure No. 5.

Because the quarks that make up the matter (atomic, planetary and galactic) travel within their own subspace of domain, the relative motion of the atomic subspace with regard at the other two quarks (planetary and galactic) generates a vector resistant that determines a torque with the maximum intensity at $45^{\circ}$-angle $\alpha$ on figure- of the equatorial plane due to the shape of the Earth (on tropic zones) and null in equator or poles, for the whole inertial mass in movement that go up or go down on its surface, generating a whirlwind or whirlpool of movement accelerated in the form of helix. In areas of land where the plane tangent to the surface is exactly $45^{\circ}$ of the equatorial plane, is where the whirlwind or whirlpool reaches its maximum intensity.

These areas match exactly with areas of violent hurricanes and tornadoes where is maximum the Coriolis Effect.

The speeds of relative movement between the subspaces of a rotating Coriolis disk -see figure above for the southern hemisphere, where $w_{\mathrm{x}}$ is the angular speed of plane $(a, r, b)$ of a rotating Coriolis disk and the points " $a$ " and " $b$ " belong to the plane $(x, y)$ - are easily calculated as shown in the following expression:

$$
v_{a}=w_{T} d_{a} \quad \text { and } \quad v_{b}=w_{T} d_{b} \quad \text { then } \quad v_{r}=w_{T}\left(d_{b}-d_{a}\right)=2 \sqrt{r^{2}+R^{2}} \operatorname{Sin}[\alpha] w_{T},
$$

where $v_{\mathrm{a}}$ is the tangential speed in point " $a$ ", $v_{\mathrm{b}}$ is the tangential speed in point " $b$ ", $v_{\mathrm{r}}$ is the tangential resulting speed plane of a rotating Coriolis disk, $w_{\mathrm{T}}$ is the angular speed at the equator of the Earth, the angle $\alpha$ is the latitude of the place where the plane $(a, r, b)$ is tangential to the surface of the Earth, " $x$ " is the axis of rotation of the Earth, " $y$ " belongs to the equatorial plane, $R$ is the distance between rotation plane $(a, r, b)$ to the center of Earth and $r$ is the radius of the plane in rotation.

Note: This phenomenon is common to all celestial body, easily observable in the Sun and Jupiter.

\section{A.2.2 Interpretation within TCS Theory of Olbers' Paradox}

Two phenomena solves this old paradox: the electromagnetic hysteresis, independently on the radiated energy of the source due to energy loss as a function of distance traveled; and as well because the human eye and all optical observation instruments detect a limited spectrum, if not, we would see the whole sky lit up.

\section{A.3 Experimental Test of Bell's Inequalities Using Time-Varying Analyzers}

This experiment was conducted by Dr. Alain Aspect in 1982.

\section{A3.1 Interpretation within TCS theory}

When the receivers (the 1/4 wavelength door absorption and the opposite end door receiver -photovoltaic cell-) uses a monochromatic light LASER on the same axis and at the same distance from the light issuer, if there is partial or total photon absorption into the door absorption, will be perceived a decrease of photons that are received in the door receiver. The reason why photons are lost on the opposite end door receiver is due to that were destroyed along the route.

Who destroyed the photons and how? The following answer is: When a photon is annulled by a magnetic field on the door absorption surface, only are absorbed the photon electromagnetic energy that is into the atomic subspace. At the same time that the photon is absorbed a gravitational wave is generated within the subspace following order (planetary-magnetic radiation phenomena, see section 8.2) with equal energy and spherical shape spread within the planetary subspace -the energy is transformed, not destroyed-. This gravitational wave has equal frequency and it is in phase with the absorbed photon and travels to a great speed, the speed of light elevated to the second power -exactly $8.98755179 \times 10^{16} \mathrm{~m} . \mathrm{s}^{-1}$. This gravitational wave with spherical shape generated by the absorbed photons destroys the photons that are aligned in their way and who are in the opposite gravitational phase, the phase of this gravitational wave generated is coherent with the phase of the electromagnetic wave of photon that is destroyed.

Therefore, if the door absorbs $100 \%$ of the photons, a certain percentage of the photons always will pass through the opposite end of the device without being destroyed, this is due to the photon destruction depends basically on the physical distance between both ends aligned with the device, the photon density per unit time consisting on cast packages and diverted one another extreme, speed of the light and speed of the gravitational waves.

This experiment is a test of two important issues:

(a) It demonstrates the existence of a class of wave that is not electromagnetic.

(b) The speed of this new class wave is enormously greater that the speed of light. 
Decreases energy depending on the distance travelled by a wave

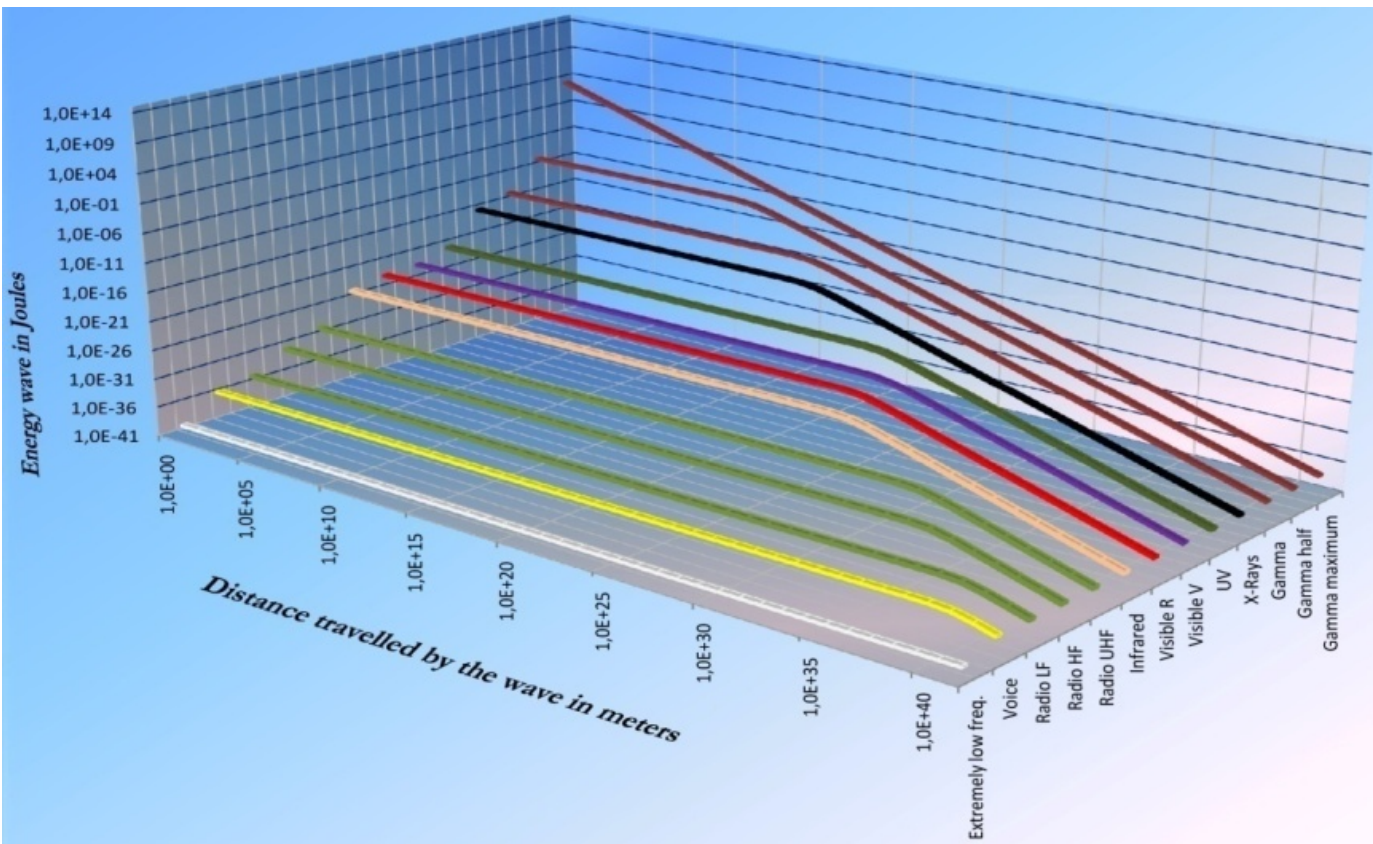

Figure 1. Decrease of electromagnetic wave energy depending on distance

Wavelength depending on the traveled distance

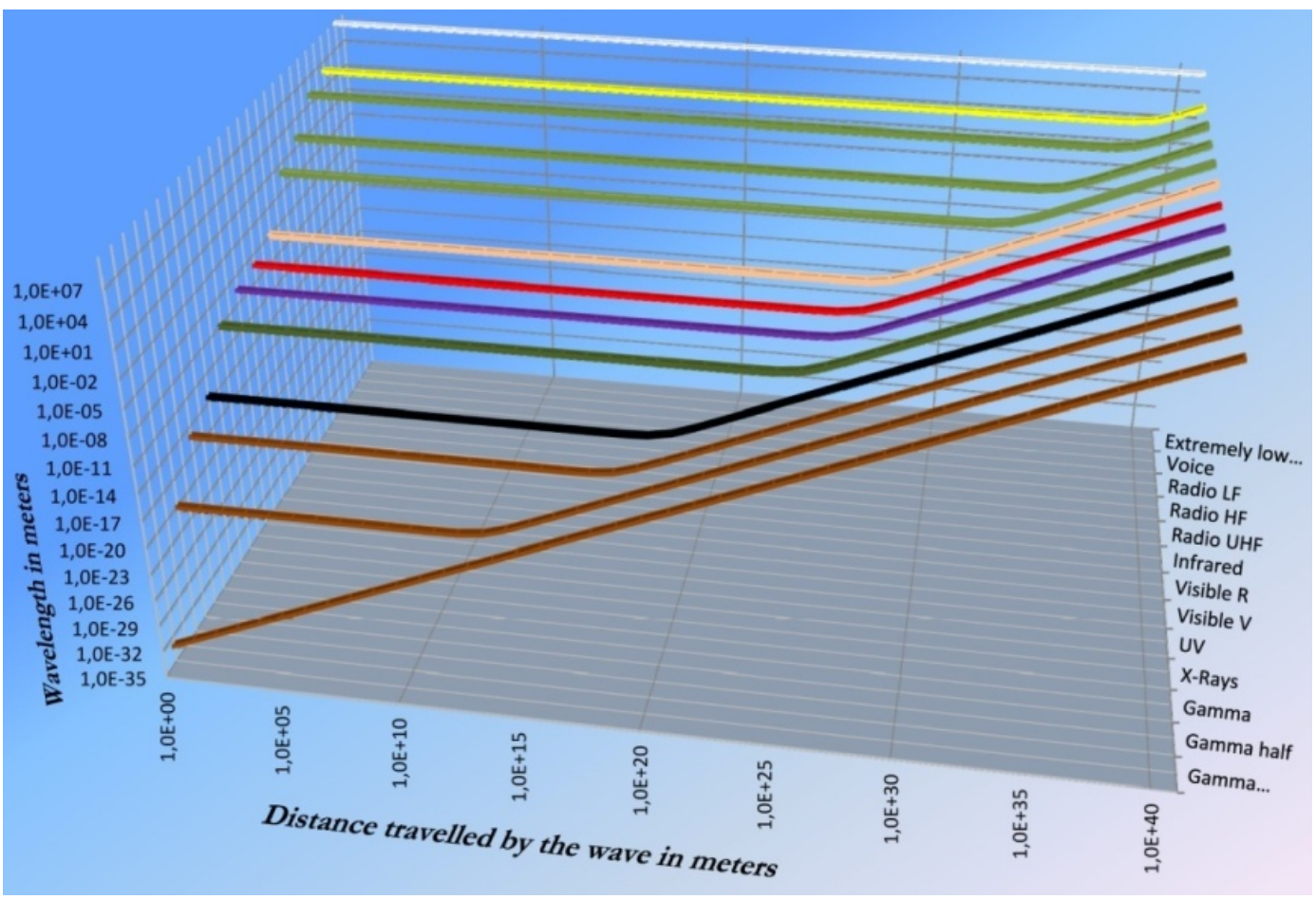

Figure 2. Increase of electromagnetic wavelengths depending on distance 
Wave Energy radiated by the sun and received by Earth

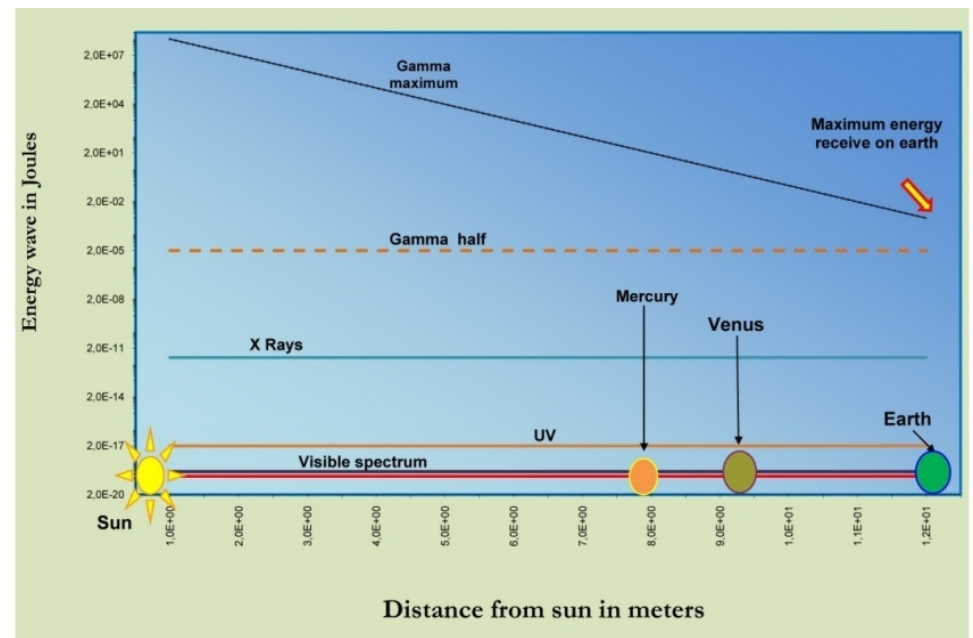

Figure 3. The maximum energy of electromagnetic waves that receives the Earth

The area of breakage of the symmetry atomic subspace on Earth

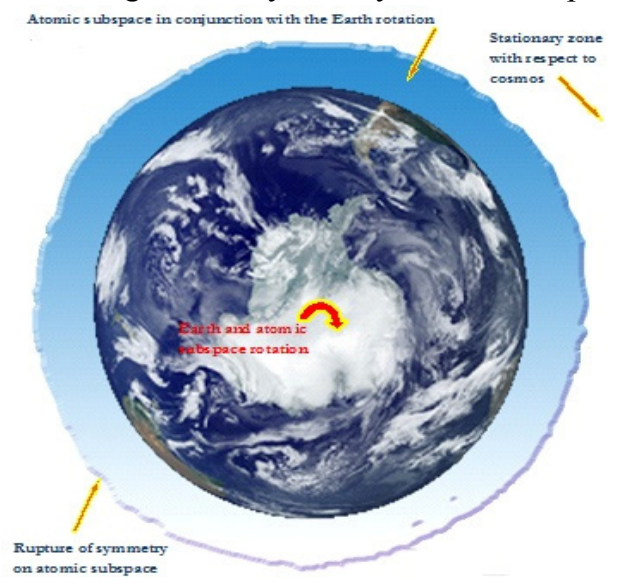

Figure 4. Satellite picture of the Earth in the Antarctic pole. Credit: NASA/ESA

The speeds of relative movement between the subspaces of a rotating Coriolis disk

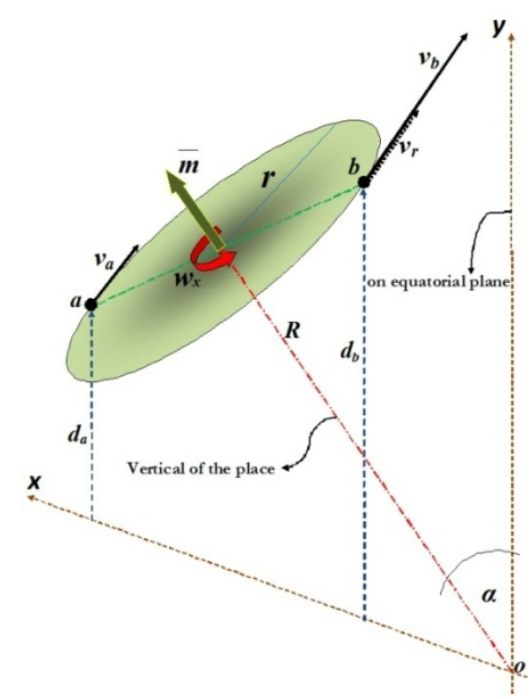

Figure 5. The rotating Coriolis disk 